\title{
Fatigue design of structures under thermomechanical loadings
}

\author{
E. CHARKALUK ${ }^{1}$, A. BIGNONNET ${ }^{2}$, A. CONSTANTINESCU ${ }^{3}$ and K. DANG VAN ${ }^{3}$ \\ ${ }^{1}$ Laboratoire de Mécanique de Lille, UMR CNRS 8107, boulevard Paul Langevin, 59655, Villeneuve d'Ascq Cedex, France, ${ }^{2}$ P.S.A Peugeot Citroen- \\ Direction de la Recherche et de l'Innovation Automobile, chemin de la Malmaison - 91 570, Bievres, France, ${ }^{3}$ Laboratoire de Mecanique des Solides - Ecole \\ Polytechnique, 91128 Palaiseau, France
}

\begin{abstract}
AB STRACT This paper presents a global approach to the design of structures that experience thermomechanical fatigue loading, which has been applied successfully in the case of cast-iron exhaust manifolds. After a presentation of the design context in the automotive industry, the important hypotheses and choices of this approach, based on a thermal 3D computation, an elastoviscoplastic constitutive law and the dissipated energy per cycle as a damage indicator associated with a failure criterion, are first pointed out. Two particular aspects are described in more detail: the viscoplastic constitutive models, which permit a finite element analysis of complex structures and the fatigue criterion based on the dissipated energy per cycle. The FEM results associated with this damage indicator permit the construction of a design curve independent of temperature; an agreement is observed between the predicted durability and the results of isothermal as well as non isothermal tests on specimens and thermomechanical fatigue tests on real components on an engine bench. These results show that thermomechanical fatigue design of complex structures can be performed in an industrial context.
\end{abstract}

Keywords dissipated energy; fatigue; thermomechanics; viscoplasticity.

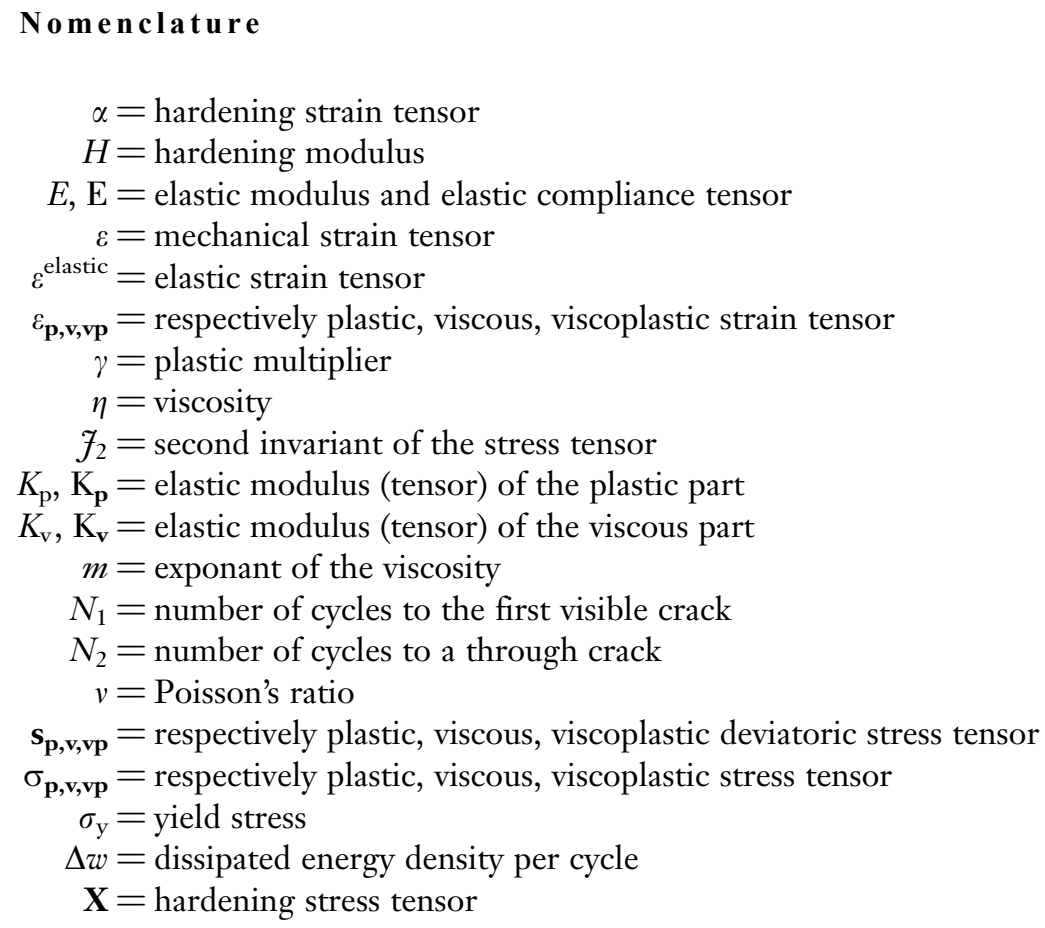

Correspondence: E. Charkaluk, Laboratoire de Mécanique de Lille, UMR CNRS 8107, boulevard Paul Langevin, 59655, Villeneuve d'Ascq Cedex, France. E-mail: eric.charkaluk@univ-lille1.fr 


\section{INTRODUCTION}

One can distinguish two principal approaches in fatigue research: the first one concerns the understanding of physical phenomena at the material scale and is the focus of a large part of the work; the second one tries to model these phenomena to establish lifetime criteria at the scale of the structure. This paper addresses the lifetime prediction of structures in the Thermomechanical Fatigue (TMF) domain for direct industrial applications with a design time reduction in mind.

In the HCF (High Cycle Fatigue) domain, a number of methods permitting the fatigue lifetime assessment of structures during the design process are now successfully used in an industrial context. A general review of methods and criteria available today was recently written by Socie and Marquis. ${ }^{1}$ We shall cite here a practical approach presented by Dang Van et $a l^{2}$ which is extensively and successfully used in the design of all components experiencing HCF by some automotive industries. ${ }^{3,4}$ We should point out that even if the choice of the criterion is important, the determination of the limit conditions and also of an equivalent fatigue loading obtained for example by the SSIA (Stress- strength Interference Analysis) ${ }^{5}$ is essential for the design approach.

In the TMF design field, there are still a large number of unresolved problems. Historically, TMF design concerned principally the nuclear and the aeronautic industries. In the first one, structures are usually designed with lifetime criteria often issued for example from the work of Coffin ${ }^{6,7}$ from General Electric and used with high safety factors. A series of similar design approaches have been formalized in different codes $\left(\mathrm{R}^{8}{ }^{8}\right.$ and RCC-MR, ${ }^{9}$ ). These rules are based on the periodic monitoring of structures which permits the eventual presence of cracks to be detected. The concept of crack propagation then allows the lifetime of the structure to be related to a critical crack size and provides the opportunity to assess the integrity of the structure during a defined period.

In the aeronautic industry, for the design of turbine blades, Chaboche ${ }^{10}$ made great advances in the eighties. These structures, which can include complex cooling channels, are essentially subjected to centrifugal loads with hold times at high temperature. For these applications, he developed complex constitutive and damage laws to take into account the elastoviscoplasticity of the material behavior and the creep-fatigue interaction in the damage process and obtained interesting results.

In other industries, take for example the automotive one, design paradigms are different. Components like exhaust-manifolds, cylinder heads or cylinder blocks have a complex geometry and the thermomechanical loading includes different phases (binding, preloading and thermal cycles) which induce 3D stress-strain distributions. The main objective is not just safety, but also lifetime prediction without intermediate monitoring. Therefore engineers need robust computational methods for the prediction of macroscopic fatigue crack initiation which must guarantee the integrity of the structures during the complete lifetime.

The first difficulty in such a design method comes from the numerical evaluation of multiaxial strains and stresses. This is due to combined thermal and mechanical loadings with variable temperatures and a large range of temperature inducing different constitutive behaviors. The second difficulty arises from the fatigue criterion itself as different damage mechanisms lead to the final failure.

In the last decades we witnessed a series of major advances in the understanding of the underlying physical phenomena and the numerical computations on structures. But to our knowledge, no global solution to this problem has been proposed with results on actual industrial structures (for a critical review and a list of unresolved problems see Chaboche, 10).

The aim of this study is to assess the lifetime of cast-iron exhaust manifolds subjected to thermomechanical loading. In this paper we propose a fatigue design method based on elastoviscoplastic constitutive models for the strain and stress computations and an energy criterion for failure. In the first section we discuss the overall items to be analyzed in a global design approach. The second section presents an overview of the constitutive behavior and of the Finite Element (FEM) computations. The experimental and computed fatigue results are discussed in the final section. This global approach leads to a reasonable lifetime prediction of actual structures and can easily be extended to other materials and structures. ${ }^{11}$

\section{GLOBAL APPROACH IN FATIGUE PROBLEMS}

The industrial design of parts generally passes through a series of iterations based on design, testing and correction. In order to reduce the number of iterations (actually about 3-5) and, implicitly, design costs and to obtain better performance of the parts, engineers are obliged to introduce predictive structural computations. Failure should be directly assessed by computations during the design. Ideally, prototype testing should not be a part of the design cycle but only a final validation of predictions in a real environment.

In a thermomechanical design process, we propose to simulate the behavior of a prototype structure under various loading conditions and to assess its lifetime. However, not all loading conditions can generally be tested. The choice of a unique representative test using the stressstrength interference analysis (SSIA) is discussed for 
example in Thomas et al. ${ }^{12}$ in the HCF context and can be extended for TMF conditions but will not be presented here.

In this work we study cast-iron exhaust manifolds. The material is a nodular graphite cast-iron with silicon and molybdenum additives.

The thermomechanical loading can be represented by heating and cooling cycles between $20^{\circ} \mathrm{C}$ and $800^{\circ} \mathrm{C}$ (approximately 1000-5000 thermal shocks depending on whether the application is a gasoline or diesel engine) combined with mechanical clamping imposed by the bolting of the hot exhaust manifold onto the colder, more rigid, cylinder head. This thermal cycle is an equivalent loading in the sense of the SSIA and should represent the same damage as the complete lifetime of the structure in the customer's vehicle. The temperature distribution is computed from the transient heat equation with boundary conditions, heat flux and heat exchange coefficients determined by a 3D transient gas flow simulation. The spatial thermal distribution is then checked by infrared thermography of a structure. More details of this crucial point of the design approach are presented by Lederer et $a l .{ }^{13}$

After the thermal loading determination, a global approach to thermomechanical fatigue design can be divided into two steps:

i A Stress-Strain Computation depending on the definition of the thermomechanical loading and the choice of the elastoviscoplastic constitutive law, and

ii A Fatigue Criterion including a damage evolution law and a failure criterion.

An important assumption is the coupling/uncoupling of the behavior and the damage. Lemaitre and Chaboche ${ }^{14}$ proposed interesting constitutive laws coupled with damage for continuum mechanics. It might therefore seem unusual to uncouple the stress-strain behavior from the damage. We accepted this hypothesis and the underlying approximations in order to be able to compute a $3 \mathrm{D}$ structure $\left(\approx 10^{5}\right.$ degrees of freedom $)$ in a reasonable computational time (some hours) with existing computers.

\section{Stress-Strain computation}

It is obvious that the mechanical behavior should be reliable at both minimum and maximum temperatures, where cast iron has plastic and viscous behavior, respectively. One can distinguish two classes of models: physical ones representing precisely the microscopic phenomena at a material scale and specifically related to a certain type of macroscopical loading: creep, relaxation,... and phenomenological ones representing a macroscopic mechanical behavior. In order to keep the final objective of a global simple representation of the material behavior at the scale of the structure, we shall restrict our choices to the second class of models.

\section{Fatigue criterion}

The second aspect concerns the fatigue criterion. Two different ways could be followed to determine a damage evolution law. The first one is the continuum damage mechanics developed in particular by Lemaitre et al. ${ }^{14}$ The second one considers parametric laws relating mechanical fields and lifetime such as the MansonCoffin law, or the Strain-Range Partioning method. ${ }^{15}$

Damage evolution laws based on continuum damage mechanics induce two particular problems. On the one hand, models are complex and calibration of the material parameters is difficult. On the other hand the damage evolution laws are often based on mean and maximal stresse ${ }^{14}$ which are not easily defined in a non isothermal cycle. For example, the often proposed ratio $\left(\sigma / \sigma_{\mathrm{u}}\right)$, where $\sigma$ can be the von Mises equivalent stress or the mean stress and $\sigma_{\mathrm{u}}$ the ultimate stress, has no physical or mechanical interpretation in this complex loading context.

Parametric laws are based on the pioneering work of Manson and Coffin ${ }^{6,16}$ with a proposed relation between cumulated plastic strains and lifetime. However, this cannot be simply extended for non isothermal multiaxial loadings due to the same lack of mechanical and physical meaning of plastic strain range in this particular context. ${ }^{17}$ This type of parametric law is interesting in an industrial context because of its simplicity but it requires an analysis of the more adapted mechanical fields in a thermomechanical context.

The last point concerns the failure criterion, which must guarantee the integrity of the structure during its lifetime. In our particular context, the design objective imposes that no macroscopic cracks should form during the lifetime. Crack growth can be separated in two stages: an initial one where the crack is confined to a small volume and does not influence the macroscopic behavior of the structure and a final one where the crack length directly influences the load distribution in the structure. This design criterion will impose the condition that the structure does not enter the final stage of cracking during its lifetime.

The objective of this study is the interpretation of FEM computations in order to obtain the critical damage zone and the lifetime of the structure.

\section{THERMOMECHANICAL FATIGUE ANALYSIS}

\section{FEM computations}

The numerical evaluations depend essentially on the choice of a constitutive law and an underlying integration algorithm. 
In order to take into account the plastic and viscous behavior at low and high temperature, respectively, elastoviscoplastic constitutive laws have been chosen. Two models have been finally selected, one classical unified viscoplastic model ${ }^{10}$ with a linear kinematic hardening:

$$
\begin{aligned}
\varepsilon^{\text {elastic }} & =\frac{1+v}{E} \sigma-\frac{v}{E} \operatorname{tr}(\sigma) \mathbf{I} \\
\sigma & =\frac{3}{2} \frac{1}{H} \mathbf{X} \\
\dot{\varepsilon}_{\mathrm{vp}} & =\frac{3}{2}\left\langle\frac{\mathcal{f}_{2}(\sigma-\mathrm{X})-\sigma_{\mathrm{y}}}{\eta}\right\rangle^{m} \frac{\mathbf{s}-\mathrm{X}}{\mathcal{f}_{2}(\sigma-\mathrm{X})}, \\
\dot{\alpha} & =\dot{\varepsilon}_{\mathrm{vp}} \\
\sigma & =\mathrm{E}:\left(\varepsilon-\varepsilon_{\mathrm{vp}}\right)
\end{aligned}
$$

and one involving an additive partition of stress into viscous and plastic components ${ }^{18}$ named two layer viscoplastic model:

$$
\begin{aligned}
\varepsilon_{\mathbf{v}}^{\text {elastic }} & =\frac{1+v}{K_{\mathrm{v}}} \sigma_{\mathbf{v}}-\frac{v}{K_{\mathrm{v}}} \operatorname{tr}\left(\sigma_{\mathbf{v}}\right) \mathbf{I} \\
\varepsilon_{\mathrm{p}}^{\text {elastic }} & =\frac{1+v}{K_{\mathrm{p}}} \sigma_{\mathbf{p}}-\frac{v}{K_{\mathrm{p}}} \operatorname{tr}\left(\sigma_{\mathbf{p}}\right) \mathbf{I} \\
\alpha & =\frac{3}{2} \frac{1}{H} \mathbf{X} \\
\dot{\varepsilon}_{\mathbf{v}} & =\frac{3}{2}\left[\frac{\mathcal{f}_{2}\left(\sigma_{\mathrm{v}}\right)}{\eta}\right]^{m} \frac{\mathbf{s}_{\mathbf{v}}}{\mathcal{F}_{2}\left(\sigma_{\mathrm{v}}\right)} \\
\dot{\varepsilon}_{\mathbf{p}} & =\gamma \frac{\mathbf{s}_{\mathrm{p}}-\mathbf{X}}{\mathcal{F}_{2}\left(\sigma_{\mathbf{p}}-\mathbf{X}\right)}, \\
\dot{\alpha} & =\dot{\varepsilon}_{\mathbf{p}} \\
\sigma_{\mathbf{p}} & =\mathrm{K}_{\mathbf{p}}:\left(\varepsilon-\varepsilon_{\mathbf{p}}\right) \\
\sigma_{\mathbf{v}} & =\mathrm{K}_{\mathbf{v}}:\left(\varepsilon-\varepsilon_{\mathbf{v}}\right) \\
\sigma_{\mathbf{p}} & =\sigma_{\mathbf{p}}+\sigma_{\mathbf{v}}
\end{aligned}
$$

The main difference between the two models is the coupling or uncoupling of the viscous and the plastic dissipation mechanisms. ${ }^{19}$ This could have an impact on the final damage law if one wished to relate the different constitutive quantities to the separate damage variables. However, as already stated, both laws are purely phenomenological and do not correspond to any mechanical behavior at a microscopic scale.

The material parameters have been identified from isothermal tension-compression-relaxation tests and are supposed to vary linearly between the test temperatures. It is obvious from this choice of constitutive models that the transient cyclic behaviour of the material, hardening or softening, has been neglected. This hypothesis has later been verified on the LCF tests and has been shown as reasonably correct.

Three numerical integration algorithms have been tested under the Abaqus Standard finite element code for the integration of the constitutive law: forward Euler scheme (explicit), backward Euler scheme with a first order series expansion (explicit), and a completely implicit backward Euler scheme with radial return. ${ }^{20}$ In the anisothermal context with a changing yield limit it turned out ${ }^{19}$ that only the backward Euler scheme with a radial return performed with reasonable convergence rates and large time steps. The time performance between the different schemes varied between 1 and 5 . The complete numerical computation for an exhaust manifold, typically $\approx 10^{5}$ degrees of freedom, took several hours on a CRAY C-90 parallel computer.

Two particular aspects can be underlined. On one hand, the comparison of the stress-strain loop obtained with both models (see on Fig. 1) of a structure shows very close mechanical responses which indicates a similar modeling of the material behavior in the structure with those different constitutive laws.

On the other hand, it is important to observe the multiaxial aspects of the stress and strain tensors. Figure 2 shows the stress-strain loops obtained on an exhaust manifold subjected to a thermomechanical loading cycle, near the surface where $\sigma_{z z}=0$.

One can remark that the other directions ( $x x$ and $y y$ ) are both submitted to high stress and strain level. In Figs 3 and $4, \sigma_{x x}$ and $\sigma_{y y}$ are compared for a thermal fatigue test specimen and for an exhaust manifold.

In the case of the specimen, the loading path is highly non proportional and for the exhaust manifold, it is non proportional but with approximately constant biaxiality ratio. Those different examples show the multiaxial mechanical responses of the different structures under transient thermal loading. It will be important to determine a fatigue criterion compatible with this type of

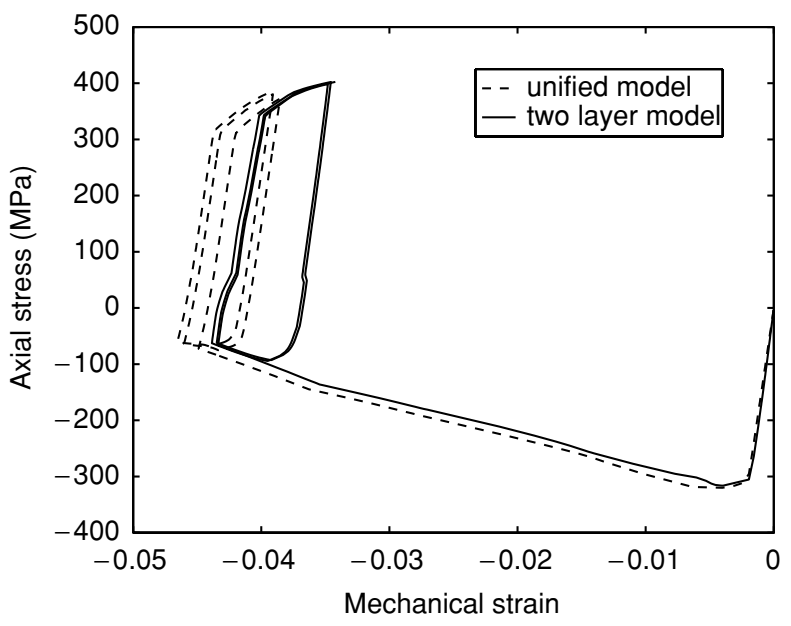

Fig. 1 Comparison of both constitutive models on the mechanical response obtained from the simulation of the thermal fatigue test. 
general loading path. The results of the previous FEM computations are the inputs of the final fatigue analysis.

\section{Fatigue criteria}

In order to obtain a multiaxial fatigue criterion, independent of the temperature (this will be illustrated in the next part of the paper), we have chosen the dissipated energy per cycle as a damage indicator:

$\Delta w(x)=\int_{\text {cycle }} \sigma(x, t) \cdot \varepsilon(x, t) \mathrm{d} t$,

where $\sigma, \varepsilon$ denote the stress and strain tensor fields, respectively, and $x$ the current analyzed point of the structure.

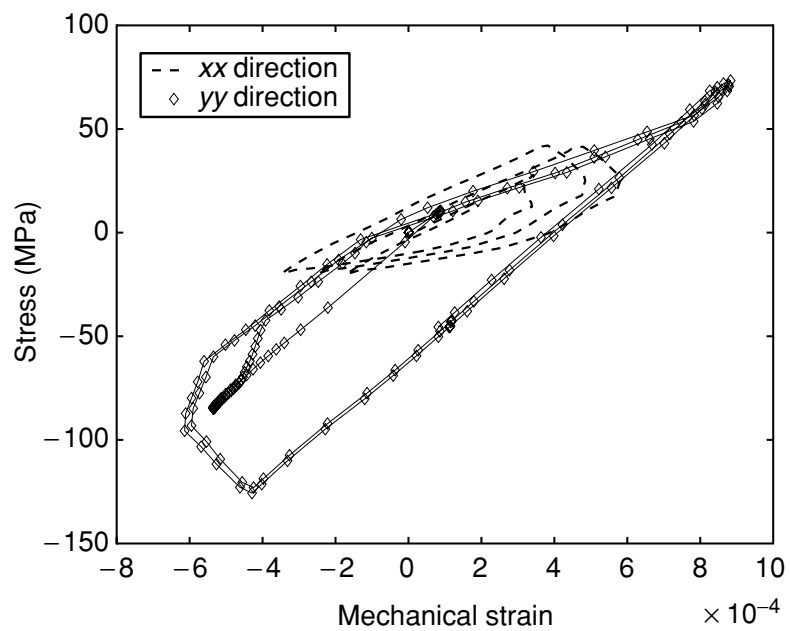

Fig. 2 Stress-strain loops obtained in $x x$ and $y y$ directions on an exhaust manifold, near the surface where $\sigma_{z z}=0$ shows the multiaxial response of the structure.

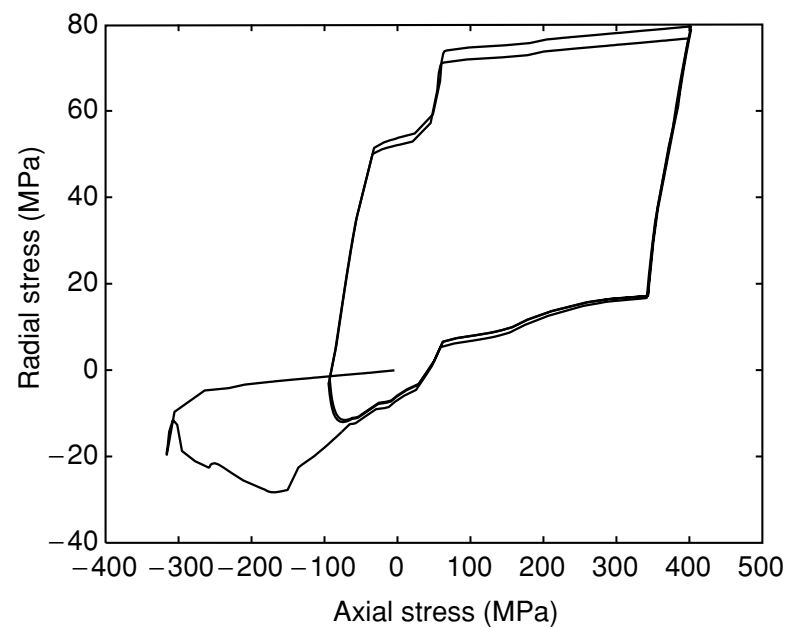

Fig. 3 General non proportional loading path obtained on a thermal fatigue test specimen.
This damage indicator should be interpreted as an upper bound of the energy dissipated in damage and thus of damage itself, as justified in a more general approach by Feltner and Morrow ${ }^{21}$ or Luong. ${ }^{22}$

A precise analysis of the relationship between cyclic behaviour and dissipated energy per cycle has been provided in a series of papers by Skelton. ${ }^{23,24} \mathrm{He}$ showed on one hand that the cumulated dissipated energy at the saturation point (see Fig. 5) can be considered as a material constant expressing the necessary energy to propagate the crack within the size of the process zone. On the other hand he related the lifetime of the specimen to a crack propagation law driven by the dissipated energy per cycle.

Reinterpreting Skelton's results we can assume that the cycle at $N_{\text {sat }}$ represents the cyclic behaviour of the material and that $N_{f}$ represents the failure of the elementary volume at the structure scale. A relation between the

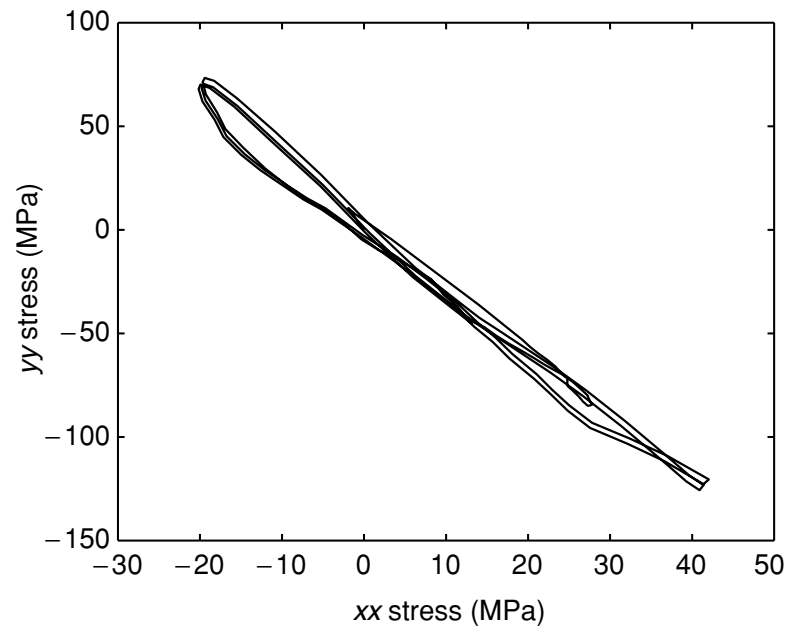

Fig. 4 Loading path obtained in the case of thermal shock on an exhaust manifold.

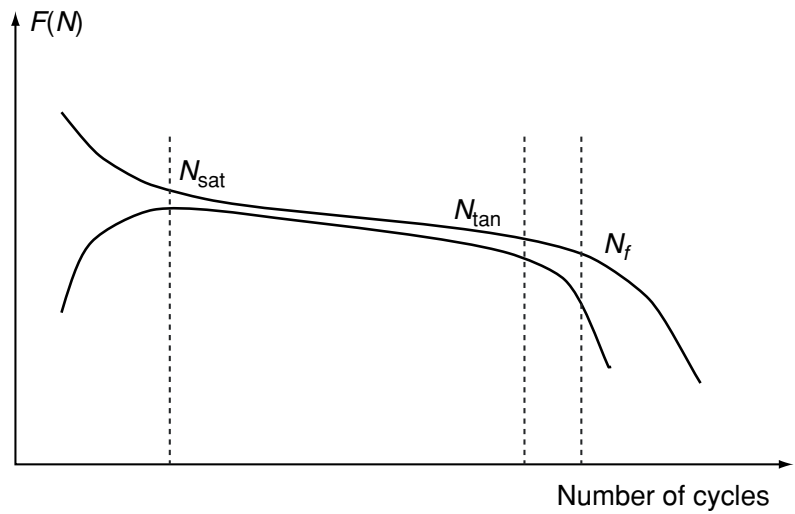

Fig. 5 Schematic representation of the cyclic behavior of the material, ${ }^{23,24}$ 
dissipated energy per cycle and the number of cycles to failure has been identified. A detailed discussion of these points can be found in Ref. [17].

\section{FATIGUE EXPERIMENTS AND RESULTS}

\section{Tests conditions}

Three different fatigue tests were performed. Let us recall the main features of the tests (for more details, see: ${ }^{17}$ Isothermal strain controlled LCF tests at $R_{\epsilon}=-1$ at different temperatures between $350^{\circ} \mathrm{C}$ and $700{ }^{\circ} \mathrm{C}$. The strain rate was $10^{-3} \mathrm{~s}^{-1}$ and three different total strain ranges were tested: $\pm 0.25 \%, \pm 0.5 \%, \pm 1 \%$. Some tests were performed at $700^{\circ} \mathrm{C}$ with a dwell at the maximum strain in the tensile or compressive part of the cycle. Thermal fatigue tests on clamped specimens, heated by the Joule effect. The maximum temperature varied between $40^{\circ} \mathrm{C}$ and $700^{\circ} \mathrm{C}$ with a heating rate of $20^{\circ} \mathrm{C} \mathrm{s}^{-1}$. The maximum temperature was obtained in the center of the specimen with an axial thermal gradient of $30^{\circ} \mathrm{C}$ $\mathrm{mm}^{-1}$. The test parameters are the clamp value $(183000 \mathrm{~N} / \mathrm{mm}$ to $227000 \mathrm{~N} / \mathrm{mm})$ and the dwell time at $700{ }^{\circ} \mathrm{C}(60 \mathrm{~s}$ and $900 \mathrm{~s})$ : Thermomechanical tests were performed on an engine bench for three different exhaust manifolds geometries. The maximum temperature varied between $20^{\circ} \mathrm{C}$ and $700^{\circ} \mathrm{C}$. It is important to note that the temperature distribution is not uniform in the structure as some areas are never warmer than $200^{\circ} \mathrm{C}$. The heating rate was approximately $10^{\circ} \mathrm{C} \mathrm{s}^{-1}$.

\section{Experimental and computed results}

In the case of the isothermal LCF tests, the dissipated energy per cycle corresponds to the area of the hysteresis loop and is determined from the experimental results. The failure criterion corresponds conventionally to a load drop of $10 \%$. Figure 6 shows the relatively temperature independence of the results which permits the construction of the fatigue criterion.

In the case of the thermal fatigue tests, no local strain measurement was possible due to the axial thermal gradient which induced a strain gradient in the middle of the specimen. Therefore, FEM simulations of the Joule effect heating and of the thermomechanical loading have been performed using the elastoviscoplastic constitutive laws presented before. The maximum values of the dissipated energy per cycle and the corresponding areas were determined from these computations for each loading case. The failure criterion is the same as in the isothermal LCF tests. The number of cycles to failure is then determined experimentally.

The same method is applied to the thermomechanical tests on exhaust manifolds. FEM computations were

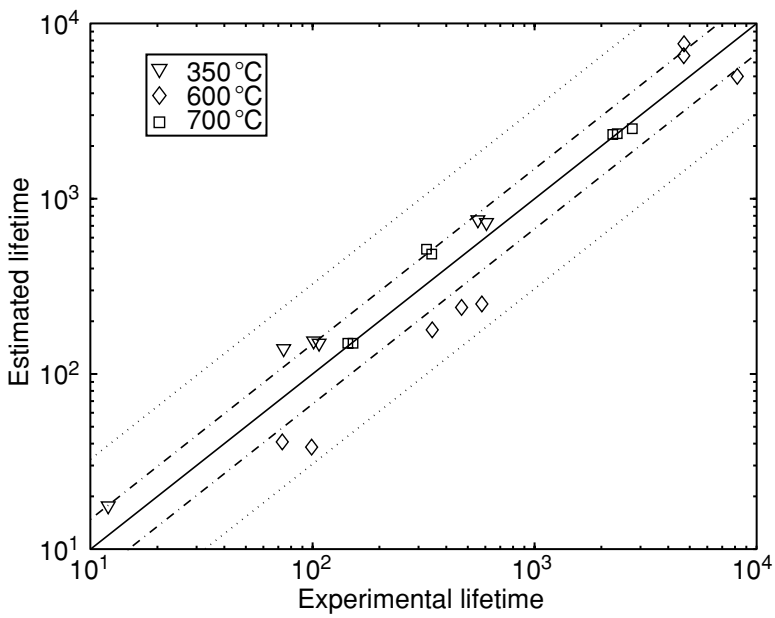

Fig. 6 Comparison of experimental and estimated lifetime obtained on LCF tests at three different temperatures. This shows the relative independence of the criterion to the temperature. The dashed lines correspond to one and three standard deviation.

performed. The thermal computation corresponds to the simulation of the engine bench loading. A thermomechanical computation using the same elastoviscoplastic constitutive law is then performed with this thermal loading and the clamping force of the exhaust manifold on the cylinder head. The maximum values of the dissipated energy per cycle and the corresponding areas were determined from those computations. Then, as no load drop measurement is possible, two definitions of the number of cycles to failure could be proposed:

$N_{1}$ - first visible crack,

$\mathrm{N}_{2}$ - through crack.

As already stated, before $N_{1}$ cycles, the crack can be accepted as it is small with regards to the structure -, i.e. it has no influence on macroscopic mechanical fields. After $N_{1}$ cycles, it leads, in all cases, to the failure of the components. The failure criterion is therefore based on $N_{1}$.

The fatigue results are presented on the Fig. 7. The isothermal LCF experimental dissipated energy per cycle and the associated number of cycles to failure determined the mean line and the standard deviation, corresponding to a factor of \pm 3 on the lifetime in the case of three standard deviations.

One can remark that the scatter of the data independent of temperature is reasonably low. In the case of thermal fatigue tests, the computed dissipated energy per cycle leads to a good prediction of the lifetime comparing to the observed experimental lifetime. This proves the possible generalization of the dissipated energy per cycle as a multiaxial thermomechanical fatigue criterion. As a consequence of these reliable predictive results, this method is now systematically applied in the engine design department as soon as the geometry is well defined and permits 
a very good prediction of possible TMF cracks. This point has been verified with real tests on an engine bench.

The maximum value of the dissipated energy per cycle indicates the crack initiation area and can be compared with those observed experimentally, see Figs 8 and 9 . This type of predictive result was obtained for each computed exhaust manifold (see Fig. 7). One can remark that those areas do not correspond systematically to the areas of maximum temperature or maximum cumulated plastic strain. ${ }^{12}$

These results demonstrate that the different hypothesis and simplifications about uncoupling mechanical behavior and damage seem to be sufficient for this type of TMF design. This was also illustrated by Verger et al. ${ }^{11}$ in the case of aluminium alloys cylinder heads and Sermage $e t a l .{ }^{25}$ for biaxial steel specimens.

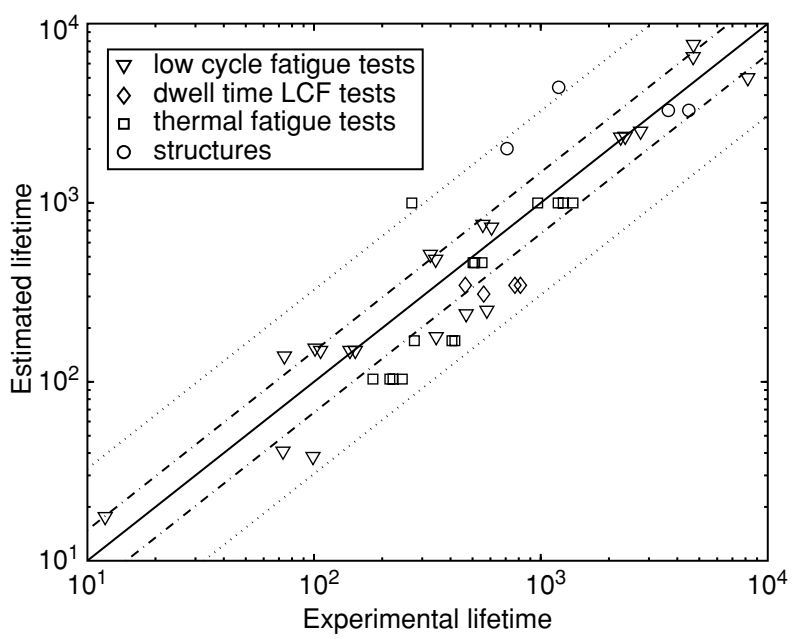

Fig. 7 Comparison between experimental and predicted lifetimes (in cycles). The dashed lines correspond to one and three standard deviation.

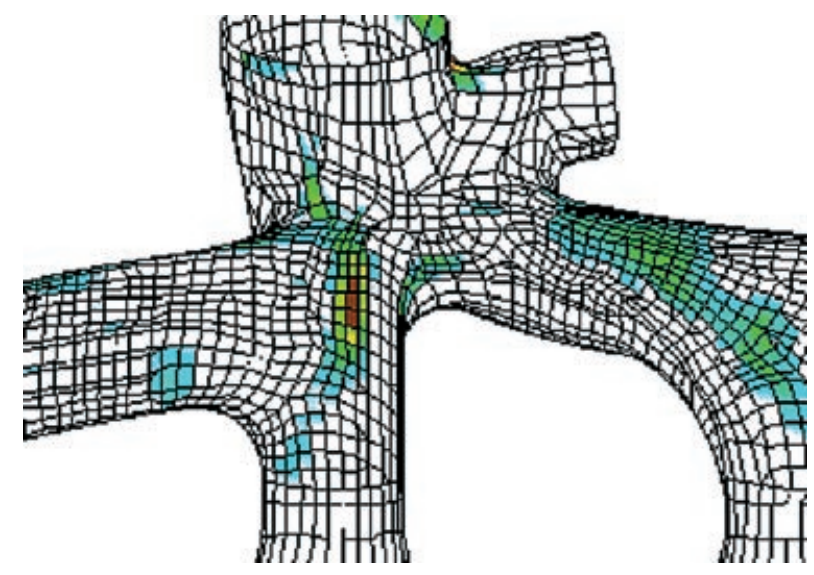

Fig. 8 Computation of a prototype exhaust manifold: predicted cracked area (inner shell).

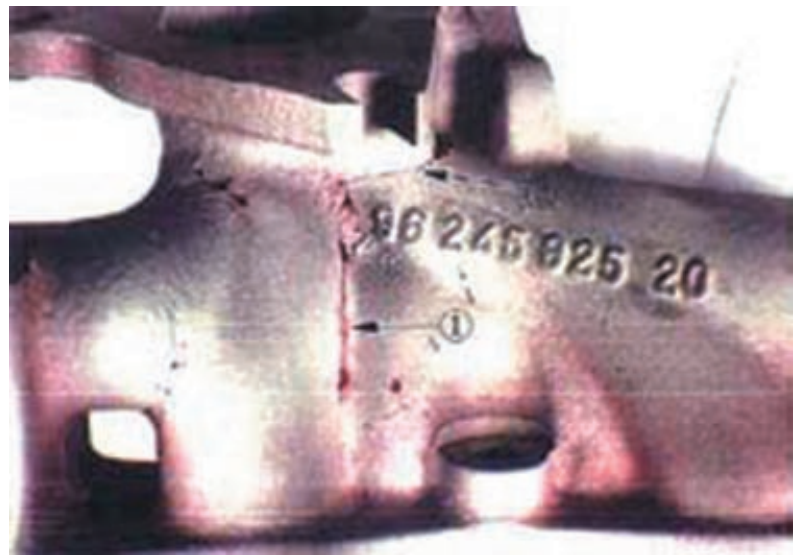

Fig. 9 Results of an engine bench test: cracked area observed on the prototype.

A particular aspect of the influence of the mean stress on lifetime has not been taken into account in this fatigue approach and is well known for its importance on damage. In this study, it seems to have had a negligible impact. In a more general case, it would be possible to define a mean stress in a variable temperature context. This would mean replacing the well known $\left(\sigma_{m} / \sigma_{u}\right)$ ratio used by most authors with well defined intrinsic parameters. This will necessitate more specific tests and studies. ${ }^{26}$

\section{CONCLUSION}

A global approach to design for structures subjected to thermomechanical fatigue loading has been applied successfully to the case of cast-iron exhaust manifolds. This approach is based on a thermal $3 \mathrm{D}$ computation, an elastoviscoplastic constitutive law and the dissipated energy per cycle as a damage indicator associated with a failure criterion. This permits a design curve independent of the temperature to be obtained.

This approach was developed in an anisothermal, multiaxial context corresponding to the analyzed structures and is applicable to other materials and structures. A key feature of such an approach remains the knowledge of the thermomechanical loading, in particular the transient temperature distribution on the structure. A series of problems regarding the choice of simple constitutive models and their impact on 3D structural computation in TMF context as well as the corresponding damage laws are still unresolved.

\section{Acknowledgements}

The authors would like to thank Guy Lederer (PSA) for the thermal computations and the fruitful discussions and Denis Bertheau and Franck Morel at L.M.P.M. (ENSMA-Poitiers, France) for the LCF tests on specimens. 


\section{REFERENCES}

1 Socie, D. F. and Marquis, G. P. (2000) Multiaxial Fatigue. S. A. E. International. pp.

2 Dang-Van, K. (1999) Introduction to fatigue analysis in mechanical design by the multiscale approach. In: High-Cycle Fatigue in the Context of Mechanical Design, CISM courses and lectures no. 392. Springer, pp. 1691-1710.

3 Bignonnet, A. (1996) Fatigue design in automotive industry. In: Fatigue '96, 6th International Fatigue Congress, (Edited by G. Lutjering and H. Nowack) pp. 1825-1836.

4 Vaillant, E., Berard, J. Y., Lienard, C. and Royer, C. (1999) Methodologie de dimensionnement en endurance des pieces de train. Paris. (18eme journees de Printemps de la SF2M.)

5 Thomas, J. J. and Bignonnet, A. (1999), L'equivalence fatigue: etat de l'art. (18eme journees de printemps, SF2M.)

6 Coffin, L. F. (1953) Study of the effects of cyclic thermal stresses on a ductile material. Trans. ASME. 53 A76: 931-950.

7 Coffin, L. F. (1973) Fatigue at high temperatures. In: Fatigue at Elevated Temperatures. ASTM STP. 520, pp. 5-34.

8 Ainsworth, R. A. (1996) Assessment Procedure for the High Temperature Response of Structures. Issue 2. Technical Report. Nuclear Electric Ltd, Barnwood UK.

9 RCC-MR. (1985) Technical Appendix a3, Section 1, Subsection Z. and Materials Design and Construction Rules for Mechanical Components of fbr Nuclear Test Islands. Technical report, AFCEN, Paris.

10 Chaboche, J. L. (1997) La Fatigue des Materiaux et des Structures. Hermes, pp. 617-644.

11 Verger, L., Constantinescu, A. and Charkaluk, E. (2002) Thermomechanical fatigue design of aluminium components. Temperature-Fatigue Interaction. ESIS Publication 29, L. Rèmy and J. Petit, Eds. Elsevier Sc. pp. 309-318.

12 Thomas, J. J., Perroud, G., Bignonnet, A. and Monnet, D. (1998) Fatigue design and reliability in the automotive industry. In: Fatigue Design '98. Third International Symposium on Fatigue Design. (Edited by G. Marquis). pp. 1-11.

13 Lederer, G., Charkaluk, L., Verger, E. and Contantinescu, A. (2000). Numerical lifetime assessment of engine parts submitted to thermomechanical fatigue, application to exhaust manifold design. SAE Technical Paper Series, 2000-01-0789.
14 Lemaitre, J. and Chaboche, J. L. (1985) Mecanique des Materiaux Solides. Dunod.

15 Halford, G. R., Hirschberg, M. H. and Manson, S. S. (1973) Temperature effects on the strain range partitioning approach for creep fatigue analysis. In: Fatigue at elevated temperature ASTM STP 520, pp 658-669.

16 Manson, S. S. (1953) Behaviour of Materials Under Conditions of Thermal Stresses. Technical Report, TN 2933, N.A.C.A.

17 Charkaluk, E. and Constantinescu, A. (2000) Energetic approach in thermomechanical fatigue for silicon molybdenum cast-iron. Mater. High Temp. 17, 373-380.

18 Kichenin, J. (1992) Comportement thermomecanique du polyethylene - application aux structures gazieres. Phd Thesis. Ecole Polytechnique, France.

19 Charkaluk, E., Verger, L., Constantinescu, A., Lederer, G. and Stolz, C. (1999) Lois de comportement viscoplastiques anisothermes pour calculs cycliques sur structures. In: Actes $d u$ 4eme colloque national en calcul des structures. (Edited by D. Guedra-Degoerges, Pl. Ladeveze and M. Raous). Teknea, pp. $575-580$.

20 Simo, J. C. and Hughes, T. J. R. (1998). Computational Inelasticity. Springer.

21 Feltner, C. E. and Morrow, J. D. (1961) Micro-plastic strain energy hysteresis energy as a criterion for fatigue fracture. Trans. ASME (60-MET-2), 1-11.

22 Luong, M. P. (1995) Infrared thermographic scanning of fatigue in metals. Nucl. Engng. Des 158, 363-376.

23 Skelton, R. P. (1991) Energy criteria for high temperature low cycle fatigue. Mat. Sci. Technol. 7, 427-439.

24 Skelton, R. P. (1993) Cyclic hardening, softening and crack growth during high temperature fatigue. Mat. Sci. Technol. 9, 1001-1008.

25 Sermage, J. P., Lemaitre, J. and Desmorat, R. (2000) Multiaxial creep-fatigue under anisothermal conditions. Fatigue Fract. Engng Mater: Struct. 23, 241-252.

26 Verger, L., Charkaluk, E. and Constantinescu, A. (2001) A macroscopic crack initiation criterion in multiaxial thermomechanical fatigue. Proceedings of the 6th International Conference on Biaxial and Multiaxial Fatigue and Fracture, M. de Freital, Ed. IST Lisbon. pp. 867-874. 